\title{
Guillaume Sire
}

Centre d'analyse et de recherche interdisciplinaires sur les médias Institut français de presse

- Université Paris 2 - Panthéon Assas

\section{Cinq questions auxquelles Google n’aura jamais fini de répondre}

C'est devenu un réflexe pour l'immense majorité d'entre nous. Dès qu'on ne sait pas, au moindre doute, on se tourne vers Google. Le moteur répond. Le moteur répond toujours. Oracle moderne, pythie algorithmique. Interface simple et rassurante. Il y a beaucoup de gens et beaucoup de choses dans l'arrière-boutique, mais on préfère que ce soit apparemment simple. On préfère que ce soit rassurant. Google nous sert à la fois de guide, de boussole et de carte. Le dispositif scanne, indexe et hiérarchise les documents accessibles sur le Web. Il braque son projecteur et décide qui aura droit à la lumière et qui, au contraire, sera condamné à la figuration ou aux coulisses de l'espace numérique. Il nous prévient : «ce contenu, pour vous, maintenant, à l'endroit où vous êtes, est sans doute plus pertinent que cet autre contenu, lui-même probablement plus pertinent que cet autre contenu, qui lui-même est pertinent, mais moins, etc.»

Il n'est pas étonnant, et à vrai dire plutôt rassurant, qu'une telle activité soit controversée. Si une technologie prétend traiter l'information à l'échelle du monde, il est prévisible qu'elle soit accusée, à tort ou à raison, de ne pas la traiter comme il faudrait. Cela est d'autant plus vrai qu'on se trouve avec le moteur Google dans un contexte où, d'une part, on ne sait pas exactement comment il fonctionne, et où, d'autre part, son propriétaire jouit d'une position de quasi-monopole dans la plupart des pays du monde.

Parmi les nombreuses controverses dont l'entreprise Google fait l'objet, nous nous intéresserons ici en particulier à cinq débats concernant le traitement de l'information effectué par le moteur. Nous ne prétendrons pas que ces controverses constituent des problèmes qu'il serait nécessaire et urgent de résoudre, mais plutôt qu'elles sont consubstantielles au moteur, en cela qu'elles contribuent à en faire ce qu'il est, c'est-à-dire un dispositif sociotechnique d'information et de communication qui n'est pas, et ne sera jamais, parfaitement satisfaisant du point de vue de chacun des acteurs que son action concerne. Les ingénieurs 
chargés de veiller au bon fonctionnement du moteur, et de le faire évoluer, sont confrontés à ces cinq controverses au quotidien. Ils ne peuvent ni les nier ni les résoudre, et doivent composer avec elles en effectuant certains détours, certaines concessions, certains ajustements. C'est pourquoi nous disons que ces controverses contribuent à faire du moteur ce qu'il est effectivement, plutôt que ce que ses concepteurs (ou n'importe qui d'autre) auraient voulu qu'il soit idéalement. Elles font partie de Google. N'ayant pas vocation à être résolues, elles en feront toujours partie. Aussi est-il préférable de les connaître si l'on veut prendre avec les pincettes qu'il convient les résultats que le moteur nous retourne lorsque nous lui adressons nos requêtes.

\section{Neutralité du moteur}

L'une des principales controverses concernant le traitement de l'information effectué par Google est liée à la perception du rôle joué par un moteur de recherche : est-il un objet neutre qui devrait être parfaitement objectif ou est-il par nature biaisé et subjectif? La première façon de voir consiste à considérer que le moteur est un relais passif (Pasquale, 2006; Chandler, 2008). Dans cette perspective, il devrait traiter les documents de la même façon, et ne jamais privilégier une source ou une opinion par rapport à d'autres sources et d'autres opinions. Le principal défaut de ce point de vue est de suggérer qu'un moteur ne devrait pas hiérarchiser les contenus (Grimmelmann, 2014).

Le point de vue opposé consiste à considérer le moteur comme un éditeur actif, subjectif, responsable de l'énoncé qu'il produit au même titre que les organes de presse, et protégé comme eux aux États-Unis par le premier amendement de la Constitution (Goldman, 2006; Volokh et Falk, 2012). La liste de résultats exprimerait dans ce cas le seul point de vue des concepteurs du moteur, ces derniers ne pouvant pas être attaqués sous prétexte qu’un document a été privilégié.
Ceux qui considèrent le moteur comme un outil neutre mettent en avant l'aspect technique du classement des documents, tandis que ceux qui considèrent que c'est un éditeur soulignent son caractère socialement construit. Les premiers se concentrent sur ce que le moteur fait, les seconds sur ce qu'il dit (Grimmelmann, 2014).

Selon James Grimmelmann (Ibid.), le caractère inconciliable des deux arguments tient au fait que le premier (qu'il nomme "théorie du tuyau») considère l'activité de Google du point de vue des éditeurs de contenus tandis que le deuxième (qu'il nomme "théorie de l'éditeur») se positionne du point de vue du concepteur du moteur. La relation entre le moteur et les contenus est ainsi présentée sous l'angle binaire : conduit ou éditeur, objectif ou subjectif, technique ou humain. Grimmelmann s'étonne du fait que le point de vue de l'utilisateur ne soit, lui, jamais, ou pas assez, considéré, et propose une troisième voie. En se plaçant du point de vue de l'utilisateur, il suggère que Google est un "conseiller» dont la qualité dépend essentiellement de sa capacité à garantir l'accès à l'information et à faire preuve de loyauté en ne considérant pas dans le calcul algorithmique d'autres intérêts que ceux de l'internaute.

\section{Intervention manuelle}

Il est courant d'entendre un éditeur se plaindre, à tort ou à raison, d'avoir été sanctionné manuellement par les équipes de Google. Même si l'entreprise a clamé le contraire pendant longtemps, on sait désormais qu'il peut arriver qu'un site soit «blacklisté» manuellement. Cependant on ignore quelles sont les conditions exactes pouvant conduire à une telle sanction, et quelle est la fréquence des interventions manuelles. Certains observateurs pensent que Google aurait raison de punir les fermes de contenus ${ }^{1}$ créées dans le but de tromper son algorithme. Selon ce même type d'argument, Google 
aurait raison de sanctionner manuellement les sites pornographiques, révisionnistes, etc. L’argument opposé est avancé par les partisans de la neutralité du moteur, selon lesquels il ne devrait pas être du ressort des employés de Google de choisir quels contenus doivent être référencés. Les partisans de cet argument craignent une forme de censure et défendent l'idée selon laquelle le moteur n'est qu'un «tuyau».

Récemment, il a été révélé que des travailleurs à domicile, employés par les entreprises Leapforce, Butler Hill et Lionbridge, jouaient un rôle dans le processus de sélection et de hiérarchisation. Un manuel de 160 pages est transmis à des évaluateurs, à qui il est demandé de donner une des appréciations suivantes à chacun des sites visités : «Vital», «Useful», « Relevant », «Slightly Relevant », «OffTopic or Useless» ou «Unratable». Un autre indicateur est renseigné manuellement : le «Page Quality». Pour cet indicateur, les évaluateurs doivent juger de la réputation du site : bonne, mauvaise, ambiguë, $\mathrm{OK}$, malicieuse ou impossible à déterminer.

Nous ignorons quel est l'impact de ce travail manuel sur la liste de résultats. Cependant, il est intéressant de considérer que Google peut être conduit à faire "exactement le contraire de son programme d'action» en procédant à une évaluation manuelle, ce qui, de fait, limite «l'automatisme de l'algorithme» (Farchy et Méadel, 2013).

La controverse demeure. À quel point faut-il intervenir manuellement? Pour quel motif? Comment? Est-ce que le fait que les ingénieurs de Google puissent intervenir manuellement ne contribue pas à leur conférer une forme de responsabilité éditoriale lorsqu'un contenu illégal apparaît dans les résultats? La controverse semble d'autant plus insoluble que Google ne publie pas les détails de l'algorithme qui prévaut à la hiérarchisation des documents, et qu'il est par conséquent impossible de prouver empiriquement la bonne foi de ses porte-parole lorsque ceux-ci prétendent qu'aucune intervention manuelle n'a eu lieu.

\section{Transparence de l'algorithme}

À part la poignée d'ingénieurs qui l'ont conçu, personne ne connaît les critères et les pondérations qui prévalent au calcul de pertinence des documents ${ }^{2}$. Dès le début des années 2000 , certains observateurs ont fait savoir qu'il était d'après eux aberrant qu'un algorithme censé hiérarchiser l'information et organiser le savoir à l'échelle du monde puisse faire l'objet d'un secret industriel (Brown et Duguid, 2000; Introna et Nissenbaum, 2000).

Les fondateurs de Google, qui au moment de la création du moteur avaient pourtant appelé de leurs vœux un algorithme transparent (Brin et Page, 1998), ont décidé de ne plus dévoiler en détails les critères et les pondérations de leur formule. Cela leur permet de compliquer la tâche des éditeurs mal intentionnés, qui essayent de piéger le moteur pour donner de la visibilité à des documents non pertinents. Si l'algorithme était connu de tous, ce genre de pratique risquerait en effet de se multiplier et les utilisateurs du moteur seraient les premiers punis. La controverse découlant de cette absence de transparence, relativement nécessaire pourtant, est essentiellement d'ordre épistémologique : Google propose de répondre à toutes les questions du moment qu'aucun internaute ne lui demande comment son moteur parvient à réaliser un tel tour de force. Comme dit le philosophe Paul Mathias (2009), «c'est un peu comme si savoir exigeait qu'on ne sût pas pourquoi ni comment l'on sait!» La controverse est également d'ordre économique : si l'algorithme était publié, les concurrents de Google risqueraient de s'en inspirer pour concevoir leurs propres algorithmes. Toutefois, contrairement à un secret industriel comme la recette du Coca-Cola, le problème avec Google tient au fait qu'il ne s'agit pas de produire une boisson désaltérante mais un dispositif de communication. Ne pas publier l'algorithme, c'est empêcher les internautes de réfléchir aux critères qui ont prévalu à la hiérarchisation des informations (i.e. les empêcher de comprendre et de critiquer la méthode). 
La controverse perdure. Google prétend, à juste titre, que si l'algorithme était transparent alors les résultats risqueraient d'être moins pertinents, tandis que ses opposants prétendent qu'il est impensable d'utiliser un dispositif qui prétend organiser la connaissance sans avoir la possibilité de savoir sur quels critères une telle organisation est établie. C'est dans le cadre de ce débat que Jean-Noël Jeanneney (2005) a suggéré la mise en place d'un algorithme européen transparent, qui pourrait être utilisé «en connaissance de cause et aussi critiqué, pour des améliorations éventuelles, par qui le voudrait».

\section{Incitations au favoritisme}

Selon certains économistes, la volonté pour Google de répondre au mieux à la requête d'un utilisateur peut s'opposer à la volonté de maximiser son profit (Hagiu et Jullien, 2011; Taylor, 2013). Notamment, les dirigeants de l'entreprise peuvent avoir intérêt à privilégier dans les résultats du moteur leurs propres sites plutôt que les sites des services concurrents. Par exemple, si l'internaute formule la requête "Paris Brest itinéraire», Google a intérêt à privilégier dans ses résultats le lien vers le service Google Maps plutôt que vers les services ViaMichelin ou Mappy. De la même façon, si l'internaute cherche un comparateur de prix, Google a intérêt à le conduire vers Google Shopping plutôt que Twenga. C'est pourquoi certains des concurrents de Google sont très virulents à l'encontre de la firme, l'accusant d'utiliser la position dominante de son moteur de recherche généraliste pour se livrer à une concurrence déloyale sur le marché de la recherche spécialisée en référençant mieux ses propres services que les services de ses concurrents. C'est cette accusation qui a valu à Google de faire l'objet mi-avril 2015 d'une communication de griefs lancée par la Commission européenne, qui pourrait lui coûter une amende de $10 \%$ de son chiffre d'affaires, soit environ 6 milliards de dollars.
En outre, Google est une régie publicitaire qui aide les éditeurs à monétiser leurs contenus grâce à des publicités provenant de son réseau d'annonceurs. Les éditeurs peuvent ainsi nouer un partenariat avec Google, grâce auquel la firme s'occupera pour eux de trouver des publicités pertinentes à afficher près de leurs contenus. Les revenus de ces publicités seront partagés entre l'éditeur et Google. Économiquement parlant, Google a donc intérêt à privilégier ses partenaires dans les résultats de son moteur, en plus de ses propres services (Rieder et Sire, 2014; 2015).

Puisque l'algorithme n'est pas connu, il est impossible de savoir si Google (dont les porte-parole jurent que ce n'est pas le cas) n'a pas tendance à privilégier dans les résultats du moteur généraliste ses propres sites ou bien les sites d'entreprises partenaires de son activité d'intermédiation sur le marché publicitaire. Car comme l'ont dit les fondateurs de Google eux-mêmes, en l'absence de transparence, il serait tout à fait possible «d'ajouter un petit facteur dont le but serait d'avantager les entreprises "amies" dans les résultats, et soustraire ce même facteur aux entreprises concurrentes. Ce type de biais serait extrêmement difficile à détecter mais n'en aurait pas moins un impact significatif sur le marché» (Brin et Page, 1998).

Finalement, l'observateur Dan Sullivan considère qu'il existe un conflit d'intérêts fondamental dans la situation de Google, et se demande dans quelle mesure une entreprise qui délivre un important trafic aux éditeurs peut également leur fournir les moyens de monnayer ce trafic (Sullivan, 2009). L'économiste Alexander White (2013) s'étonne quant à lui, à la lecture des différentes enquêtes menées par les autorités de la concurrence, du silence qui entoure le fait que la firme Google soit manifestement incitée à manipuler les résultats de son moteur - «on se demande s'il n'y aurait pas un éléphant dans la pièce» sourit-il. 


\section{Personnalisation des résultats}

Google personnalise les résultats de son moteur de recherche. Autrement dit, deux internautes qui effectuent la même requête pourront obtenir des résultats très différents en fonction de ce que le moteur sait à leur sujet. Cela induit une forme de "tension dialectique» entre individualisation et standardisation (Miconi, 2014). Bien qu'elle soit vue par certains observateurs comme une solution au problème de biais systématique inhérent au fonctionnement du moteur, et qu'elle puisse aboutir à des résultats plus pertinents pour l'utilisateur (Goldman, 2006), la personnalisation soulève des débats houleux.

Le principal argument qui s'y oppose consiste à pointer du doigt le risque d'enfermer un internaute dans sa propre culture, en l'empêchant d'être confronté à d'autres pensées que la sienne (Sunstein, 2002; Van der Hof et Prins, 2008). Dans le cas d'une personnalisation très forte, le moteur deviendrait un outil de confirmation plutôt qu'un outil d'information : il conforterait l'internaute dans un savoir préexistant à l'expression de son besoin d'information lieu de lui ouvrir de nouveaux horizons (Bozdag, 2013).

Eli Pariser (2011) a largement contribué à la publicité de cette controverse en expliquant dans un ouvrage très commenté comment il était possible que Google maintienne l'internaute à l'intérieur d'une «bulle-filtre» informationnelle. Selon lui, un internaute à propos duquel le dispositif aurait identifié une tendance progressiste se verrait proposer davantage de contenus progressistes, ou seulement des contenus progressistes. Il ne serait pas, ou peu, dérangé par des avis contraires au sien. Eli Pariser y voit une forme de gatekeeping dont le danger est qu'elle peut conduire à une forme subtile de censure. C'est pourquoi il préconise que des outils soient développés qui permettraient à l'utilisateur, quel que soient ses connaissances techniques, de contrôler facilement le degré de personnalisation des résultats produits par le moteur de recherche lorsqu'il effectue une requête.

Certains auteurs ne sont pas d'accord avec Eli Pariser. C'est le cas par exemple du sociologue Dominique Cardon (2011), d'après qui l'idée selon laquelle la personnalisation «réduit l'univers de l'utilisateur à ses propres centres d'intérêt ne se vérifie guère. Internet contribue moins à isoler les internautes dans un espace informationnel restreint qu'à renforcer la coupure entre ceux qui s'exposent massivement aux informations et ceux qui s'éloignent de plus en plus de l'espace public».

Le flou nimbant les modalités de fonctionnement du moteur rend difficile le fait de savoir à quel point les résultats sont personnalisés, et si oui comment, selon quels critères. C'est pourquoi le débat perdure entre les partisans et les détracteurs de la personnalisation des résultats produits par Google. Malgré les difficultés méthodologiques que représente la mesure du degré de personnalisation, certaines études empiriques ont néanmoins été tentées, révélant que les résultats de Google n'étaient pas aussi personnalisés qu'on pourrait le croire à la lecture des travaux d'Eli Pariser (Von Schoultz et Van Niekerk, 2012).

Par ailleurs, une partie du débat concernant la personnalisation porte sur l'identité du bénéficiaire de ces techniques. Si certains auteurs pensent que la personnalisation peut profiter aux internautes (Goldman, 2005), d'autres affirment qu'elle aurait tendance à profiter aux annonceurs à qui Google offre de cibler les utilisateurs en fonction des intérêts identifiés (Fuez et al., 2011). Un tel biais visant à favoriser les annonceurs plutôt que les utilisateurs risquerait d'amplifier la formation des bulles-filtre et pourrait contribuer à imperméabiliser leurs frontières (Granka, 2010).

Le moteur Google est un dispositif méta-éditorial. Il produit du discours à propos des discours, un regard sur des regards. Au cours des années 2000, tandis que leur entreprise s'imposait face à la concurrence, les concepteurs 
du moteur Google sont passés d'une volonté descriptive - si une page est intéressante elle figure en tête du classement - à une ambition normative - si une page figure en tête du classement, alors elle est intéressante (Eisermann, 2009). La firme détient aujourd'hui une position de quasimonopole dans le domaine de la recherche d'information. En Europe, plus de 90 \% des requêtes sont adressées à son moteur (AT Internet, 2015). Dans un tel contexte, Google fait l'objet de plusieurs controverses, dont certaines interrogent ce que le moteur fait à et fait de l'information. Celles que nous avons présentées ici peuvent être résumées par la série de questions suivantes :

1) Le moteur Google doit-il être neutre et objectif, ou au contraire endosser un rôle d'éditeur nécessairement subjectif?

2) Les ingénieurs doivent-ils laisser à l'algorithme le soin de trier l'information ou doivent-ils parfois intervenir manuellement?

3) Les détails de l'algorithme doivent-ils être communiqués ou au contraire gardés secrets?

4) Google favorise-t-il ses propres sites dans les résultats du moteur, et les sites de ses partenaires économiques?

\section{NOTES}

1. Les fermes de contenus sont des sites web hébergeant de nombreux contenus dont la valeur ajoutée est très faible et qui sont en général recopiés et publiés automatiquement dans le seul but d'y juxtaposer des encarts publicitaires et de réaliser ainsi des revenus à moindres frais.

2. On connaît certains critères pris en considération par Google, comme la centralité (i.e. le nombre de liens pointant vers le
5) Google doit-il personnaliser les résultats, et si oui à quel point?

Ces questions n'ont pas et n'auront jamais de réponses évidentes. Selon toute vraisemblance, elles demeureront controversées. Nous croyons qu'elles sont consubstantielles au fait de traiter l'information algorithmiquement, à l'échelle du monde, comme prétend le faire Google. En prenant connaissance de ces controverses, l'internaute pourra avoir à l'esprit les limites de l'outil qu'il est en train d'utiliser, et se faire sa propre idée quant à ce qui doit/ peut être fait de l'information à laquelle le moteur lui aura permis d'accéder. Il évitera à la fois le piège qui consisterait à prétendre que «le moteur est irréprochable, il faut l'utiliser les yeux fermés » et celui qui consisterait à dire qu'il ne faut plus jamais l'utiliser. Si la connaissance de ces controverses permet de rester vigilant, elle permet également de comparer le moteur Google et ses concurrents : l'utilisateur peut se renseigner pour savoir comment tel ou tel moteur répond à chacune des questions énumérées ci-avant, et faire son choix étant donné ce qu'il pense lui-même de ce que devrait être la réponse à chacune de ces questions.

document), la performance (i.e. la vitesse de chargement de la page) ou les signaux sociaux (le nombre de «like» sur Facebook et le nombre de tweets). Toutefois, on ne connaît pas la pondération de ces critères dans l'algorithme, d'une part, et d'autre part, on ne sait pas quels autres critères sont pris en considération (il y en aurait entre 200 et 300 selon les spécialistes). 


\section{RÉFÉRENCES BIBLIOGRAPHIQUES}

AT INTERNET, Baromètre des moteurs de recherche, 2015.

BozDAG, E., «Bias in Algorithmic Filtering and Personalization», Ethics and Information Technology, vol. 15, $\mathrm{n}^{\circ} 3,2013$, p. 209-227.

BRIN, S. et PAGE, L., «The Anatomy of a Large-Scale Hypertextual Web Search Engine", Computer Networks and ISDN Systems, vol. 30, n' 1-7, 1998, p. 107-117.

Brown, J. S. et Duguid, P., The Social Life of Information, Boston, Harvard Business School Press, 2000

CARdon, D., «L'ordre du Web», Médium, n² 29, 2011, p. 191-202.

Chandler, J. A., «A Right to Reach an Audience : An Approach to Intermediary Bias on the Internet», Hofstra Law Review, vol. 35, $\mathrm{n}^{\circ} 3,2008$, p. 1095-1138.

EISERmann, M., Comment fonctionne Google?, Grenoble, Université Joseph Fourier, s. e., 2009. En ligne sur : <www.igt.uni-stuttgart. de/eiserm/enseignement/google.pdf $>$, consulté le 9/9/2015.

FARCHY, J. et MÉADEL, C., «Moteurs de recherche et référencement : chassez le naturel... », Hermès, $n^{\circ}$ 66, 2013, p. 147-154.

Fuez, M., Fuller, M. et Stalder, F., «Personal Web Searching in the Age of Semantic Capitalism ", First Monday [en ligne], vol. 16, $\mathrm{n}^{\circ} 2$, 2011. En ligne sur : <firstmonday.org/article/view/3344/2766>, consulté le 9/9/2015.

Goldman, E., "Search Engine Bias and the Demise of Search Engine Utopianism", Yale Journal of Law \& Technology, vol. 8, 2006, p. 188-200.

GrankA, L. A., "The Politics of Search : A Decade Retrospective», The Information Society, vol. 26, $\mathrm{n}^{\circ}$ 5, 2010, p. 364-374.

Grimmelmann, J., «Speech Engines», Minnesota Law Review, $n^{\circ} 98,2014$, p. 868-952.

Hagiu, A. et JUllien, B., "Why Do Intermediaries Divert Search? », RAND Journal of Economics, vol. 42, nº 2, 2011, p. 337-362.

Introna, L. et Nissembaum, H., "Shaping the Web : Why the Politics of Search Engines Matters", The Information Society, vol. $16, n^{\circ} 3,2000$, p. 169-185.
JeAnneney, J.-N., Quand Google défie l'Europe: Plaidoyer pour un sursaut, Paris, Mille et une nuits, 2005.

Mathias, P., Qu'est-ce que l'Internet?, Paris, Vrin, coll. «Chemins philosophiques», 2009.

MiconI, A., «Dialectic of Google», in KöNIG, R. et RAsCH, M. (dir.), Society of the Query Reader. Reflections on Web Search, Amsterdam, Institute of Network Cultures, 2014, p. 30-40.

PARISER, E., The Filter Bubble: How the New Personalized Web Is Changing What We Read and How We Think, Londres, Penguin Books, 2011.

PASQUAle, F., «Rankings, Reductionism, and Responsibility», Seton Hall Public Law Research Research Paper, $n^{\circ} 888327,2006$. En ligne sur : <ssrn.com/abstract=888327>, consulté le 9/9/2015.

PASQUALE, F., «Internet Nondiscrimination Principles : Commercial Ethics for Carriers and Search Engines", Seton Hall Public Law Research Research Paper, $\mathrm{n}^{\circ} 1134159,2008$. En ligne sur : <ssrn.com/ abstract=1134159>, consulté le 9/9/2015

RIEDER, B. et SIRE, G., «Conflicts of Interest and Incentives to Bias : A Microeconomic Critique of Google's Tangled Position on the Web", New Media \& Society, vol. 16, n² 2, 2014, p. 195-211.

SiRE, G. et Rieder, B., «Dans les ramures de l'arbre hypertexte. Analyse des incitations générées par l'opacité du moteur Google» French Journal for Media Research [en ligne], $\mathrm{n}^{\circ}$ 3, 2015. En ligne sur : <frenchjournalformediaresearch.com/index.php?id=468>, consulté le 9/9/2015.

Sullivan, D., «KinderStart Becomes KinderStopped in Ranking Lawsuit Against Google», Search Engine Watch [en ligne], 2006. En ligne sur : <searchenginewatch.com/sew/news/2058241/ kinderstart-becomes-kinderstopped-in-ranking-lawsuit-againstgoogle $>$, consulté le 9/9/2015.

Sullivan, D., «Fights in the Google Monopoly Debate Miss Key Points", Search Engine Land [en ligne], 8 mai 2009. En ligne sur : $<$ searchengineland.com/fights-over-the-google-monopoly-misskey-points-18916>, consulté le 9/9/2015.

Sunstein, C. R., Republic.com, Princeton, Princeton University Press, 2002. 
Guillaume Sire

TAYLOR, G., «Search Quality and Revenue Cannibalization by Competing Search Engines», Journal of Economics \& Management Strategy, vol. 22, n 3, 2013, p. 445-467.

VAN DER HoF, S. et Prins, C. «Personalisation and its Influence on Identities, Behaviour and Social Values", in HiLdebrandT, M. et Gutwirth, S. (dir.), Profiling the European Citizen : Crossdisciplinary Perspectives, Dordrecht, Springer, 2008, p. 111-127.

VOLOKH, E. et FALK, D. M., «Google First Amendment Protection for Search Engine Search Results ", The Journal of Law, Economics and Policy, vol. 8, n ${ }^{\circ} 4,2012$, p. 883-900.
VON Schoultz, D. et VAN Niekerk, J., «You According to Google : the Effects of Filter Bubbling", Proceedings of the 14th annual conference on World Wide Web applications, Cape Town, Cape Peninsula University of Technology, 2012.

White, A., «Search Engines : Left Side Quality Versus Right Side Profits », International Journal of Industrial Organization, vol. 31, 2013, p. 690-701. 